\title{
A Rendezvous Node Selection and Routing Algorithm for Mobile Wireless Sensor Network
}

\author{
Yifan $\mathrm{Hu}^{1,2,3,4^{*}}$, Yi Zheng ${ }^{1,2,3}$, Xiaoming $\mathrm{Wu}^{4}$, Hailin $\mathrm{Liu}^{1,2,3}$ \\ ${ }^{1}$ Institute of Oceanographic Instrumentation, Qilu University of Technology (Shandong Academy of Sciences), \\ Qingdao, China \\ [e-mail: huyif12@163.com] \\ ${ }^{2}$ Shandong Provincial Key Laboratory of Ocean Environmental Monitoring Technology, Qingdao, China \\ ${ }^{3}$ National Engineering and Technological Research Center of Marine Monitoring Equipment, Qingdao, China \\ ${ }^{4}$ Qilu University of Technology (Shandong Academy of Sciences), Shandong Computer Science Center (National \\ Supercomputer Center in Jinan), Shandong Provincial Key Laboratory of Computer Networks, Jinan, China \\ *Corresponding author: Yifan Hu
}

Received August 10, 2017; revised January 19, 2018; revised May 6, 2018; accepted May 31, 2018; published October 30, 2018

\begin{abstract}
Efficient rendezvous node selection and routing algorithm (RNSRA) for wireless sensor networks with mobile sink that visits rendezvous node to gather data from sensor nodes is proposed. In order to plan an optimal moving tour for mobile sink and avoid energy hole problem, we develop the RNSRA to find optimal rendezvous nodes (RN) for the mobile sink to visit. The RNSRA can select the set of RNs to act as store points for the mobile sink, and search for the optimal multi-hop path between source nodes and rendezvous node, so that the rendezvous node could gather information from sensor nodes periodically. Fitness function with several factors is calculated to find suitable RNs from sensor nodes, and the artificial bee colony optimization algorithm (ABC) is used to optimize the selection of optimal multi-hop path, in order to forward data to the nearest RN. Therefore the energy consumption of sensor nodes is minimized and balanced. Our method is validated by extensive simulations and illustrates the novel capability for maintaining the network robustness against sink moving problem, the results show that the RNSRA could reduce energy consumption by $6 \%$ and increase network lifetime by $5 \%$ as comparing with several existing algorithms.
\end{abstract}

Keywords: Wireless sensor network, mobile sink, tour plan, rendezvous node, artificial bee colony

A preliminary version of this paper appeared in WCSE 2016, June 17-19, Tokyo, Japan. This version includes a complete design of proposed algorithm and supporting implementation results on MWSNs. This work was supported in part by the National Nature Science Foundation of China (No. 61401257, 61501282, 61772309), Key Project of the National Nature Science Foundation of China (No. 61332015), Shandong Provincial Natural Science Foundation (No. ZR2016DQ18), Shandong Provincial Natural Science Foundation (No.ZR2018MF003), National Key Research and Development Project (No. 2017YFC1403303). 


\section{Introduction}

W ireless sensor network (WSN) technology is the result of recent developments in the field of microelectronic mechanical systems (MEMS) and wireless communication technology that has resulted in the construction of small and smart sensors. Sensors are deployed in a wireless network and are used in civilian applications such as smart city, industrial control and environmental monitoring [1, 2]. WSN in these applications often produces high-bandwidth sensor data that needs to be gathered under stringent delay constraints [3, 4].

However, in previous work, mobility and communication capabilities are often underutilized, resulting in suboptimal solutions incurring unnecessarily energy hole and large latency $[5,6]$. Several studies have demonstrated the benefits of using a mobile sink to reduce the energy consumption of nodes and avoid problem of energy holes in mobile wireless sensor network [7, 8, 9]. Mobile sink can be a kind of Unmanned Aerial Vehicles (UAVs), which can fly long distance and monitor several targets as related technologies have been developed [10]. Many researchers and industry companies try to employ UAV for several real-world applications such as air quality measurement, traffic monitoring, and wildlife research, because the flying track of mobile sink can be optimized to be appropriate to their applications. With this trend, there are several studies related to cooperation of WSN and mobile sink, which is called mobile wireless sensor network.

In the mobile wireless sensor network, mobile sink moves in the sensed area to gather data, and returns to the base station before a deadline due to its limited running time, which would facilitate the expansion of the monitoring area. However, if the mobile sink traverses all the nodes during its movement, the moving tour would be too long and its energy consumption is too large. How to reduce its moving distance so as to prolong lifetime is an important problem. A method proposed by several articles to address this challenge is to deploy rendezvous nodes (RN) for mobile sink to visit [10,11,12,13]. During its moving tour, the mobile sink would visit temporarily RNs to gather data $[14,15]$. The RN would store data originated from source nodes and upload to the sink when the sink arrives. If the sensor node is not RN then forward its sensed data via multi-hop route to the nearest RN.

In this paper, we focus on the problem of finding an optimal moving tour of the mobile sink, and achieving small data delivery latency in the case of reducing energy consumption of sensor nodes.

\section{Related Work}

The problem for our work is how to deploy rendezvous nodes (select the location of the rendezvous nodes) for mobile sink to visit, so that the energy consumption of the WSN can be minimized, and also minimize the moving distance of mobile sink. There are several articles solving this problem.

In [16], to have better WSN energy saving, the authors plan rendezvous points for a mobile sink to travel by mapping sensors in a plane to a point on a Halin graph. They focus on balancing energy depletion to extend network lifetime with the use of mobile sinks and a proper travel plan. The scheme provides a protocol to plan rendezvous points for the travelling of mobile elements. In the selecting procedure, they consider the deadline and the optimal tour for mobile sink to travel those points. Using the Halin graph helps to solve rendezvous 
planning in polynomial time.

A heuristic WRP (Weighted rendezvous planning) algorithm is proposed in [11]. Here mobile-sink node only visits rendezvous nodes (RNs). The basic problem is computing a tour that visits all these RNs. If Sensor nodes are not RNs then forward its sensed data via multi hopping to the nearest RN. Each sensor node is assigned a weight depending on the hop distance from the tour and the number of data packets. Simulation results demonstrate that WRP enables a mobile sink to retrieve all sensed data within a given deadline while conserving the energy expenditure of sensor nodes.

Two efficient data-forwarding strategies for wireless sensor networks with a mobile sink which visits rendezvous points (RPs) to gather data from sensor nodes are proposed in ariticle [12], which is based on WRP. With the first proposed strategy, selections of the RP nodes and the final routing paths are based on the latest information of the already selected RP nodes. The second proposed strategy adopts a simpler RP node- and routing path-selection algorithm with the same level of complexity of an existing algorithm, we call it proposed-II algorithm.

However, the above protocols in $[11,12]$ is not suitable for the large scale sensor networks, as nodes number is larger, the complexity of RN selection optimization problem would be more difficult. Besides, in the problem of selecting RN, the WRP and proposed-II only consider small factors of distance between the alternative RN and RNs set, and the stored date number of alternative $\mathrm{RN}$, but not the remaining energy and its neighbors number, which would be not comprehensive enough.

To address the mobile sink tour planning and rendezvous node (RN) selecting problem, we develop a Rendezvous Node Selection and Routing Algorithm (RNSRA) to optimize the mobile sink tour planning method in the WSN. Our proposed method includes rendezvous node selection step, and rendezvous node routing step. The main contributions of this paper are as follows:

1) We develop a Rendezvous Node Selection and Routing Algorithm (RNSRA) to select a set of RNs and plan the sink moving tour, in which the RN can act as a store point for the mobile sink to visit. In order to search for optimal RNs, different from the above articles [11, 12], 4 factors are considered in the fitness function for RN selection: the remaining energy of the alternative RN, its neighbors' number, stored delivery packet number, and its distance to other RNs. This RN selection method could balance energy consumption and avoid the energy hole problem.

2) We propose an artificial bee colony optimization algorithm (ABC) to search for optimal multi-hop path from source node to the nearest rendezvous node (RN), which would efficiently forward data to the RN for storage, while minimizing the hops number of packet delivery path, in order to achieve the purpose of saving energy consumption.

The rest of this paper is organized as follows. Section 3 describes our mobile sink network model. Section 4 presents the proposed RNSRA algorithm in details. Section 5 evaluates the performance of the RNSRA by comparing with other protocols for WSN. Finally, the conclusion is presented in Section 6.

\section{System Model and Assumptions}

Our network model is a connected graph $G(V, E)$ [17], where $V$ is a finite set of sensor nodes and $E$ is the set of edges representing connection between these nodes. In the network model as shown in Fig. 1, $v_{s}^{i}(0<i<N)$ is the $i$-th source node, $v_{R N}^{j}(0<j<N)$ is the $j$-th rendezvous node, and $v_{\operatorname{Sink}}$ is the mobile sink. Suppose there exist $m(0<m<N-1)$ source 
nodes and one mobile sink, the source nodes $\left(v_{s}^{1}, v_{s}^{2}, v_{s}^{3} \ldots v_{s}^{6}\right)$ would route the packets through multi-hop relay node path to the RN $v_{R N}^{j}$, then $v_{R N}^{j}$ stores the packets, and sends to the mobile sink when the sink moves close to its communication range, as illustrated in Fig. 1. Sensor nodes that are not RNs forward their sensed data via multi-hop route to the nearest RN. The fundamental problem then becomes identifying the optimal RNs that the sink would visit within a given delay bound, and also planning moving tour for the sink.

Our network model is based on the following assumptions: 1) The area is covered by a large number of homogeneous sensor nodes. Sensor nodes are stationary, but the sink moves constantly with a relatively fixed speed. 2) Only mobile sink and rendezvous node need to know physical locations.

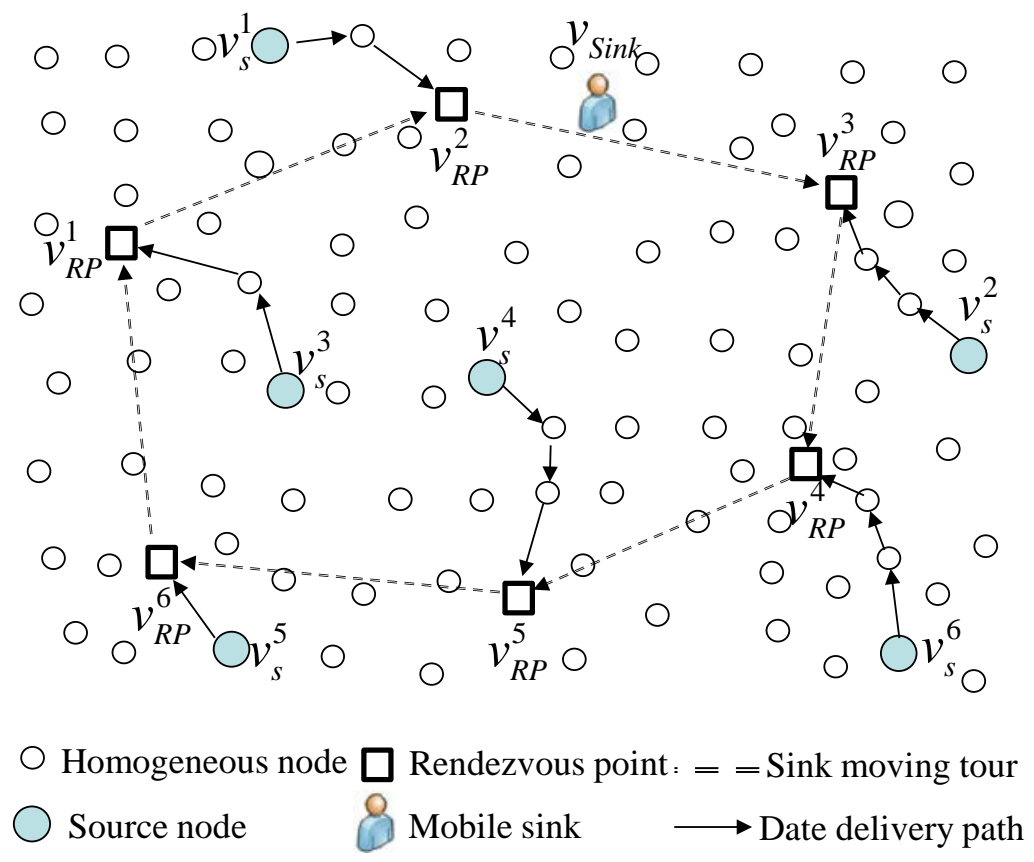

Fig. 1. The sink moving tour model for mobile WSN

\section{Design of proposed RNSRA Algorithm}

Our proposed rendezvous node selection and routing algorithm is divided into two steps: selecting rendezvous node and multi-hop routing from source nodes to rendezvous node. We firstly select several rendezvous nodes from sensor nodes, to make sure that the rendezvous nodes are evenly distributed in the network; then the multi-hop route from source nodes to the rendezvous nodes are formed, to make sure the selected route is the optimal path. The process is as below:

\subsection{Rendezvous node selection step description}

In the rendezvous node selection step of our proposed RNSRA algorithm, the RN selecting method can calculate and decide the optimal and suitable RN from the sensor nodes, in order 
to update and maintain the dynamic network route from source node to mobile sink, so that the mobile sink would plan the optimal tour and visit all these RNs.

1) Global RN (rendezvous node) selection. We define the density of RN as Dens $\left(v_{R N}\right)$, which means there would be one RN among this number of sensor nodes, then the number of RN in the network is $\operatorname{NumV}\left(v_{R N}\right)=\operatorname{Num}(V) / \operatorname{Dens}\left(v_{R N}\right)$, and the sensor node number is $\operatorname{Num}(V)$. The factors affecting the choice of RNs $v_{R N}^{i}$ contain 4 factors after normalization:

a) $\operatorname{Leng}\left(H, v_{R N}^{i}\right)$, distance rate of $v_{R N}^{i}$ to other RNs, is the ratio of the distance between $v_{R N}^{i}$ and the other selected RN set $H\left(\operatorname{Sink}, v_{R N}^{j} \ldots\right)(0<j<M a x, j \neq i)$ to the maximum length of sink moving tour $D$ :

$$
\operatorname{Leng}\left(H, v_{R N}^{i}\right)=\operatorname{Leng}\left(H, v_{R N}^{i}\right) / D
$$

b) $\operatorname{Num} P\left(v_{R N}^{i}\right)$, the packet delivery rate, is the ratio of $v_{R N}^{i}$ 's stored packet number to the max stored packet number:

$$
\operatorname{NumP}\left(v_{R N}^{i}\right)=\operatorname{Store}\left(v_{R N}^{i}\right) / \operatorname{MaxStore}\left(v_{R N}^{i}\right)
$$

c) Ene $\left(v_{R N}^{i}\right)$, the remaining energy rate, is the ratio of $v_{R N}^{i}$ 's remaining energy to its initial energy:

$$
\operatorname{Ene}\left(v_{R N}^{i}\right)=\text { Re maining Ene }\left(v_{R N}^{i}\right) / \operatorname{Initial} \operatorname{Ene}\left(v_{R N}^{i}\right)
$$

d) Neigh $\left(v_{R N}^{i}\right)$, the neighbor node rate, is the ratio of $v_{R N}^{i}$ 's neighbor nodes number to the whole network nodes number $N u m(V)$ :

$$
\operatorname{Neigh}\left(v_{R N}^{i}\right)=\operatorname{NeighNum}\left(v_{R N}^{i}\right) / \operatorname{Num}(V)
$$

The fitness function of node $\operatorname{fit}\left(v_{R N}^{i}\right)$ is:

$$
\begin{aligned}
& \operatorname{fit}\left(v_{R N}^{i}\right)=\omega_{1} \operatorname{Leng}\left(H, v_{R N}^{i}\right)+\omega_{2} \operatorname{NumP}\left(v_{R N}^{i}\right)+\omega_{3} \operatorname{Ene}\left(v_{R N}^{i}\right)+\omega_{4} \operatorname{Neigh}\left(v_{R N}^{i}\right) \\
& \text { s.t. } \quad \sum_{i=1}^{N} \operatorname{Leng}\left(H, v_{R N}^{i}\right)<D, \quad \omega_{1}+\omega_{2}+\omega_{3}+\omega_{4}=1
\end{aligned}
$$

where $\omega_{1}, \omega_{2}, \omega_{3}, \omega_{4}$ are the weights of distance rate, packet delivery rate, remaining energy rate, and neighbor node rate in the fitness function. We set $\omega_{1}=0.2, \omega_{2}=0.1, \omega_{3}=0.4$, $\omega_{4}=0.3$, which can enhance the importance of remaining energy and neighbor node number for the RN selection. $D$ is the maximum length constraint of sink moving tour for the RN $v_{R N}^{i}$.

After all the RNs are selected, our proposed algorithm would call the TSP solver to calculate a sink optimal moving tour that would visit all the RNs, so that the sink can plan the shortest distance of tour to traverse all the RNs. In our algorithm and simulation, we used a local-search-based heuristic TSP solver as mentioned in [18], in which the shortest sink moving tour would be calculated according to the RNs' position.

2) Alternative RN selection: If the remaining energy of $v_{R N}^{i}$ is less than threshold value or it is failed, one alternative RN among the one hop neighbor nodes of the failed RN would be selected, the process is the same as step 1). If suitable RN is selected, the moving tour of the mobile sink would not be changed; Otherwise, the alternative RN would be selected in the local area around the failed $\mathrm{RN}$, and the moving tour of the sink should also be changed, then the new optimal moving tour of sink is calculated and planned. 


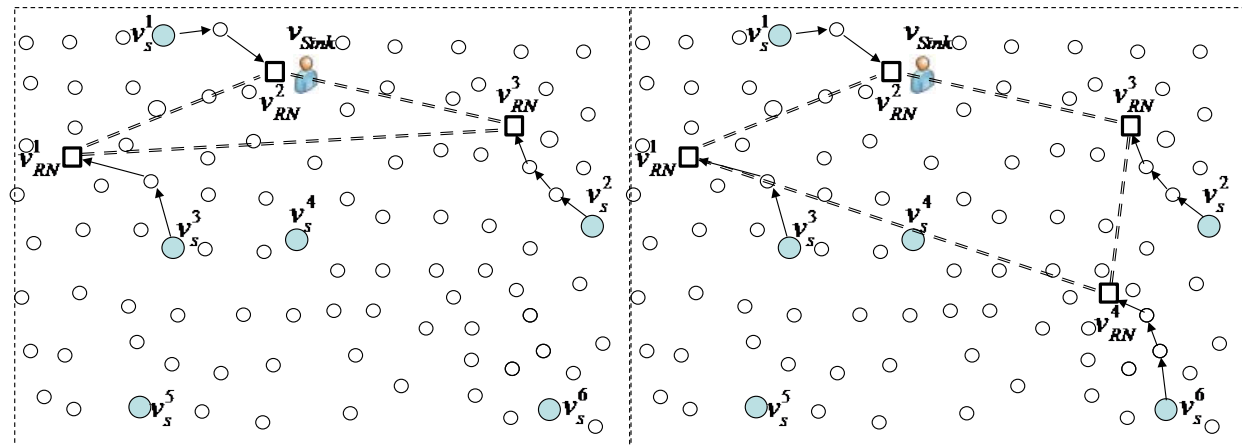

(a)

(b)

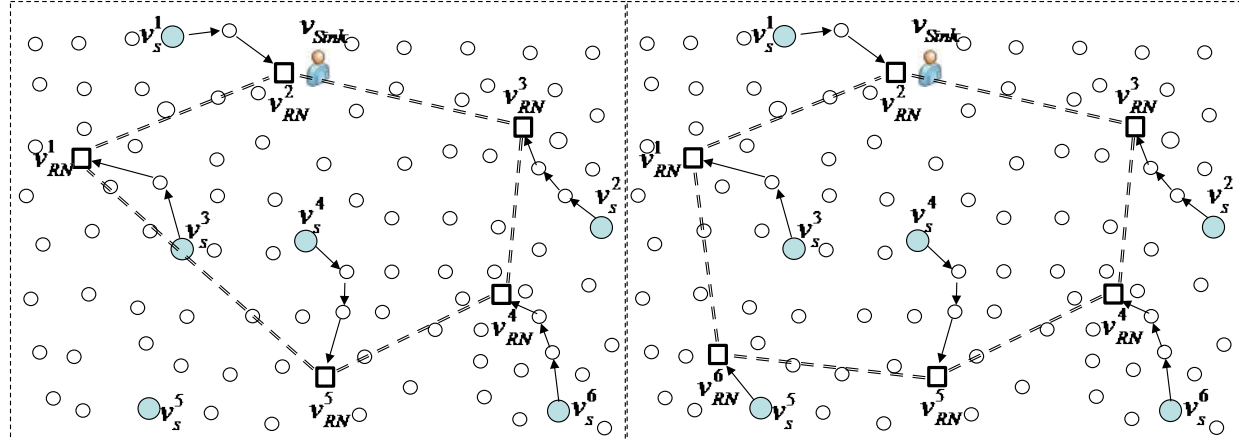

(c)

(d)

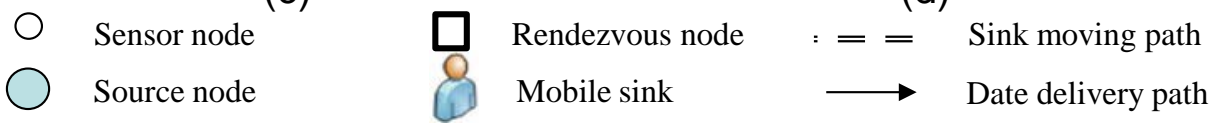

Fig. 2. The proposed Rendezvous Node Selection and Routing Algorithm process

An example in Fig. 2 shows the process of our RNSRA selecting the suitable RNs in the network. In Fig. 2 (a), the sink starts to find the third RN with optimal fitness value by the equation (5), and form the tour $\left\{v_{R N}^{2}, v_{R N}^{3}, v_{R N}^{1}\right\}$. In Fig. 2 (b), the RNSRA selects node $v_{R N}^{4}$ with the highest fitness value, and the TSP solver would return the tour $\left\{v_{R N}^{2}, v_{R N}^{3}, v_{R N}^{4}\right.$, $v_{R N}^{1}$, the length of which is less than distance $D$. Therefore, node $v_{R N}^{4}$ is added to the tour. The process continues, yielding a final tour of $\left\{v_{R N}^{2}, v_{R N}^{3}, v_{R N}^{4}, v_{R N}^{5}, v_{R N}^{6}, v_{R N}^{1}\right\}$ with a tour length $D^{\prime}$, which is less than the required tour length, else if the tour length $D^{\prime}>\mathrm{D}$, this candidate RN node would be discarded, and we would reselect another suitable RN. As shown in Fig. 2 (d), the final tour computed by our RNSRA always includes sensor nodes as RNs that have more data packets to forward, more neighbor nodes, more remaining energy, and longer suitable distance to the other RNs in the network. This method would balance the energy consumption.

Therefore, the moving tour of mobile sink is generated (such as $\left\{v_{R N}^{2}, v_{R N}^{3}, v_{R N}^{4}, v_{R N}^{5}, v_{R N}^{6}\right.$, $\left.v_{R N}^{1}\right\}$ shown in Fig. 2 (d)), and the tour plan of the mobile sink is completed. Further work is to calculate the multi-hop route between the rendezvous node and source node, so that the rendezvous node can gather information of sensor nodes periodically. 


\subsection{Rendezvous node routing step description}

In the rendezvous node routing step of RNSRA algorithm, the multi-hop route search from the source node to the rendezvous node RN will be optimized by fitness function (equation (5)), after selecting rendezvous node. We use the rendezvous node routing step of RNSRA algorithm to search the optimal data delivery route, update and maintain the multi-hop route from source node to the RN, in order to shorten the delivery path, and save network energy. The detailed routing process is as shown in Fig. 3.

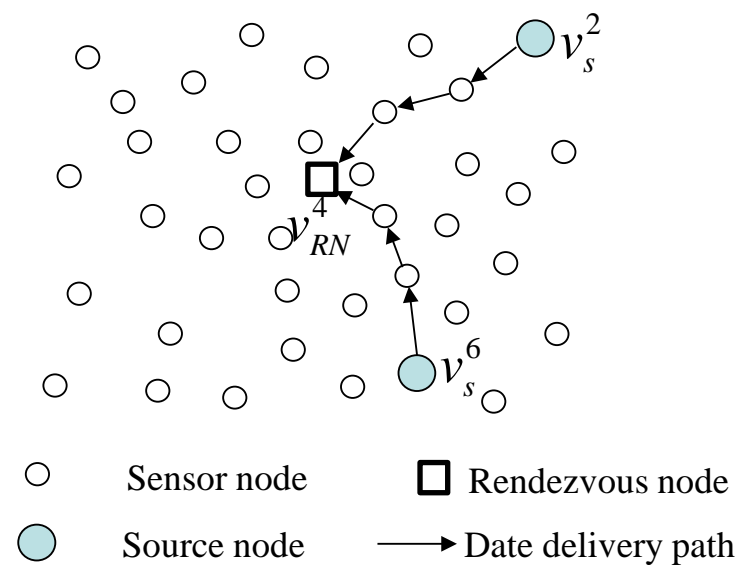

Fig. 3. The multi-hop routing process from source node to RN

\subsubsection{Searching for rendezvous node}

In this step, source node $v_{s}^{j}$ broadcasts Route Maintain (RM) packet to neighborhood nodes, in order to find new RN. The RM packet contains node battery energy $V_{b a t}\left(v_{i}\right)$ and other information. When neighbor node $v_{i}$ receives RM, it will reply Route Maintain Reply (RMR) packet and add the following information into RM: signal strength $\operatorname{RSSI}\left(v_{i}\right)$, delivery delay $\operatorname{Delay}\left(v_{i}\right)$ between its upstream neighbor node $v_{i-1}$ and itself $v_{i}$, and its battery voltage $V_{b a t}\left(v_{i}\right)$. Then it would relay packet RM to downstream neighbor node $v_{i+1}$, until all these RM packets are delivered through several multi-hop routes to the $v_{R N}$.

\subsubsection{Route optimization from source node to rendezvous node}

1) Route selection mechanism

The rendezvous node would receive totally $N$ numbers of RM packets from all these paths. Each multi-hop path $p_{j}$ from source node $v_{s}^{j}$ to the rendezvous node $v_{R N}^{i}$ would be considered as one of the optional path set $P_{\text {all }}$. Using this method, the optimal path $p_{o p}$ containing suitable relay node sequence $\left\{v_{o p}^{s}, v_{o p}^{1}, v_{o p}^{2}, \ldots, v_{o p}^{k}, \ldots, v_{o p}^{n}, v_{R N}^{i}\right\}$ would be selected from the optional path set $P_{a l l}$. The RN $v_{R N}^{i}$ would gather the information of all the nodes, and extract the information of $\operatorname{RSSI}\left(v_{i}\right)$, Delay $\left(v_{i}\right), V_{b a t}\left(v_{i}\right)$ of these nodes from $P_{a l l}$, which can be used to calculate fitness function value of path $p_{j}$. 
The facts effecting the path $p_{j}$ selection include signal strength $\operatorname{RSSI}\left(v_{i}\right)$, delivery delay $\operatorname{Delay}\left(v_{i}\right)$ between neighbor nodes, and battery voltage $V_{b a t}\left(v_{i}\right)$ of the relay node. The fitness function equation is as follow:

$$
\operatorname{fit}\left(\mathrm{p}_{\mathrm{j}}\right)=\max \left(\mathrm{k}_{1} \sum_{\mathrm{i}=1}^{\mathrm{n}} \frac{\mathrm{RSSI}_{\text {th }}}{\operatorname{RSSI}\left(\mathrm{v}_{\mathrm{i}}\right)}+\mathrm{k}_{2} \sum_{\mathrm{i}=1}^{\mathrm{n}} \frac{\text { Delay }_{\text {th }}-\text { Delay }_{\left(\mathrm{v}_{\mathrm{i}}\right)}}{\text { Delay }_{\text {th }}}+\mathrm{k}_{3} \sum_{\mathrm{i}=1}^{\mathrm{n}} \frac{\mathrm{V}_{\text {bat }}\left(\mathrm{v}_{\mathrm{i}}\right)}{\mathrm{V}_{\text {bath }}}\right)
$$

where $R_{S S I}$ is the signal strength value at threshold node $1 \mathrm{~m}$ which is $-45 \mathrm{dBm}$. Delay ${ }_{t h}$ is the end-to-end delay threshold which is set to $250 \mathrm{~ms}$. $V_{\text {batth }}$ is the node threshold battery voltage $3.5 v$. $k_{1}, k_{2}$, and $k_{3}$ are respectively the weights of distance, delay, and remaining energy constraints in the fitness function, $k_{1}=0.4, k_{2}=0.2, k_{3}=0.4$, and $k_{1}+k_{2}+k_{3}=1$. Apparently, the more suitable path selected from source to the rendezvous node would contain nodes with stronger signal strength, less delay, and higher battery voltage.

According to the fitness function equation (6), we can calculate and obtain the maximum value of fitness $\left(p_{j}\right)$ with the path $p_{j}$, and then select the optimal path $p_{\text {op }}$ with optimal fitness function value fitness $\left(p_{o p}\right)$ (such as optimal path $\left\{v_{s}^{2}, v_{1}, v_{2}, v_{R N}^{4}\right\}$ in Fig. 3).

If the optional paths number of optional path set $P_{a l l}$ is more than 5, as the selection process of calculated optional paths would be too complex, the Artificial Bee Colony intelligent algorithm (ABC) would be used to optimize the calculation of optimal path, else the optimal path is calculated by the fitness function and selected directly. Each optional path $p_{j}$ from source $v_{s}^{j}$ to $v_{R N}^{i}$ in our WSN model would be considered as a solution in the ABC algorithm. The introduction and process of $\mathrm{ABC}$ algorithm is described in the next subsection.

\section{2) Artificial Bee Colony (ABC) optimization mechanism.}

In the $\mathrm{ABC}$ algorithm, three groups of bees are generated: employed bees, scout bees, and onlooker bees. Employed bees search food sources and share the information to recruit the onlooker bees. Onlooker bees make decision to choose a food source from those found by the employed bees, and search food around the selected food source. The food source that has more nectar amount (fitness value) will have a higher probability to be selected by onlooker bees. Scout bees are transformed from some employed bees, which discard their food sources and randomly search for new ones. The position of a food source represents a potential solution. The nectar amount of a food source is the fitness value of the associate solution. The number of employed bees or onlooker bees is equal to the solutions number in the population.

In details, $X_{i}=x_{i 1}, x_{i 2}, \ldots, x_{i D}$ is the $i$-th food source (solution) in the population, $D$ is the problem dimension size. Each employed bee generates a new food source $V_{i}$ around the neighborhood of its previous food source position as follows:

$$
v_{i j}=x_{i j}+\phi_{i j}\left(x_{i j}-x_{k j}\right)
$$

where $i=1,2,3, \ldots, S N, S N$ is the population size, $j=1,2, \ldots, D$ is a random index, $x_{k}$ is a randomly selected solution in the current population $(k \neq i) . \phi_{i j}$ is a random number in the range [-1,1]. If the new $V_{i}$ is better than its parent $X_{i}, V_{i}$ would replace $X_{i}$.

After employed bees phase, the probability value $\mathrm{Pro}_{i}$ was calculated based on the fitness of the food sources (related to its nectar amount) as follow equation:

$$
\operatorname{Pro}_{i}=\frac{f i t\left(x_{i}\right)}{\sum_{i=1}^{S N} f i t\left(x_{i}\right)}
$$


where $f i t\left(x_{i}\right)$ is the fitness value of the $i$-th solution in the population, and $\operatorname{Pro}_{i}$ is proportional to $f i t\left(x_{i}\right)$. A better food source can have the higher probability to be selected.

If food source could not be improved further over a predefined number of cycles, the food source is abandoned. The value of the predefined number of cycles is another control parameter, called limit. Assume that the abandoned source is $x_{i}$, the scout bee randomly searches a new food source to be replaced with $x_{i}$. The operation is defined as follow equation:

$$
x_{i j}=x_{j}^{\min }+\operatorname{Rand}(0,1)\left(x_{j}^{\max }-x_{j}^{\min }\right)
$$

where $\operatorname{Rand}(0,1)$ is a uniformly distributed in the range $[0,1]$, and $\left[x_{j}^{\min }, x_{j}^{\max }\right]$ is the boundary constraint for the $j$-th variable.

Then if the number of iterations increases from zero to Gen, or fitness value of the solution is the optimal fitness, the optimal path $p_{o p}$ (solution) would be output. The detailed process of this proposed algorithm RNRSA is shown as below.

\section{Algorithm RNRSA. Process of route optimization \\ Input: $\quad P_{\text {all }}$ : Optional path set in network}

Gen: Algorithm Iterative number

Output: Fitness function value fit $\left(p_{o p}\right)$ of optimal route and related information

\section{Process}

: $\quad$ Source node sends the RM packet (containing optional path information) to rendezvous node through all optional paths

If Optional path number $>5$

Initialize bee colony parameter, get initial colony

For Each iterative time $T$

Calculate individual fitness, better half as employed bees, others as onlooker bees

Employed bees search and generate employed bee colony, onlooker bees search and generate new onlooker bee colony

Combine employed and onlooker bees to form iterative population and generate scout bees, update iterative colony

$T=T+1$

\section{End For}

Output optimal solution, consided as optimal path information

Else calculate optional path fitness function

$$
\text { fitness }\left(p_{j}\right)=\max \left(\left(l_{1} \sum_{i=1}^{n} \frac{R S S I_{\text {th }}}{\operatorname{RSSI}\left(v_{j}^{i}\right)}+l_{2} \sum_{i=1}^{n} \frac{\operatorname{Delay}_{\text {th }}-\operatorname{Delay}\left(v_{j}^{i}\right)}{\operatorname{Delay}_{\text {th }}}+l_{3} \sum_{i=1}^{n} \frac{V_{\text {bat }}\left(v_{j}^{i}\right)}{V_{\text {bat }_{t h}}}\right) \cdot \frac{V_{\text {bat }}\left(v_{R N}^{i}\right)}{V_{\text {bat }_{t h}}}\right)
$$

Output optimal solution 


\section{End If}

Rendezvous node would send optimal path information to the source node on path. Source node selects rendezvous node with optimal fitness, as its rendezvous node to deliver date

Algorithm Ends

\subsubsection{Rendezvous node broadcasts optimal path information to its nodes}

Once the rendezvous node has selected the optimal path $p_{o p}$ and its node sequence $\left\{v_{o p}^{s}, v_{o p}^{1}, v_{o p}^{2}, \ldots, v_{o p}^{k}, \ldots, v_{o p}^{n}, v_{R N}^{i}\right\}$ on the optimal path, it would broadcast the Route Maintaining Complete (RMC) packet to its neighbor nodes. The relay node which received RMC would check whether its ID is existed in the node set of this packet (whether this node belongs to this optimal path $p_{o p}$ ), if yes, it would add ID into the optimal path $p_{o p}$. Then it would connect its upstream node $v_{o p}^{i-1}$ with Route Maintain Complete Reply (RMRR) packet, and relay RMC to the downstream node $v_{o p}^{i+1}$, until the source node $v_{o p}^{s}$ of the optimal path received this packet. Thereafter, the optimal path construction from the source node to the rendezvous node would be completed.

\subsubsection{Overhead analysis of proposed RNSRA algorithm}

Communication overhead is always as several orders of magnitude times as the computation overhead, so the communication overhead control of our RNSRA algorithm can reflect computational complexity, and is important to reduce energy consumption. As we know, $\operatorname{Num}(V)$ numbers of sensor nodes are randomly distributed in area $\mathrm{A}$, and $\sqrt{\operatorname{Num(V)}}$ nodes are on each side, the path connecting the source and the mobile sink is assumed to be the square of the area A, which contains $\sqrt{2 N u m(V)}$ nodes. The communication radius of nodes is $r$, and the number of neighbors of a node is calculated as follows:

$$
\operatorname{NeighNum}\left(v_{i}\right)=\operatorname{Num}(V) \frac{\pi r^{2}}{A}
$$

Both the request and the response packets have the same size $S$. The communication overhead of a node generated by the packets RM, RMR, RMC, RMCR is:

$$
O=\left(3 \sqrt{2 \operatorname{Num}(V)}+\operatorname{NeighNum}\left(v_{i}\right)\right) S+4 S=\left(3 \sqrt{2 N u m(V)}+4+\operatorname{Num}(V) \frac{\pi r^{2}}{A}\right) S
$$

We can see that our algorithm generates almost the same control overhead with the proposed-II and WRP, and less than most of other conventional protocols for the WSNs.

In the network energy model [14], the equation of the node energy model is as follow:

$$
\text { ene }(l, d)=\text { ene }_{t x}(l, d)+\text { ene } r x=\left(a_{11}+a_{2} d^{n}\right) l+a_{12} l
$$

where $d$ is the distance from the node to the next-hop node, ene $e_{t x}(l, d)$ and ene $e_{r x}(l)$ are the energy consumption of sending and receiving $l$ bits of data, $n$ is the channel attenuation index, 
$a_{11}, a_{2}$ and $a_{12}$ are energy consumption parameters of sending circuit, sending amplifier and receiving circuit. Using this model, the energy consumption of the data delivery can be calculated in the simulation.

\section{Experimental Evaluation of the Protocol}

\subsection{Model and Assumption}

We evaluated our RNSRA (rendezvous node selection and routing algorithm) with simulation-based experiments, the simulation was conducted in an environment based on Matlab 2008a. The experimental hardware environments are Intel®i7-4600M, 2.90GHz CPU and $4 G B$ memory, operating system is MS windows 7 . The whole network is simulated in the area of $3500 \mathrm{~m} \times 3500 \mathrm{~m}$. The field is static and 30 300 sensor nodes are deployed uniformly, in which $10 \%$ sensor nodes are source nodes. The density of RN in the network is $\operatorname{Dens}\left(v_{R N}\right)=25$, and the mobile sink speed is $2 \sim 4 \mathrm{~m} / \mathrm{s}$. All sensor nodes have fully charged battery with $150 \mathrm{~J}$ of energy, the source node delivers packets at the rate of 20 data packets per round, with $10 \mathrm{~KB}$ of each packet size. The whole experiment will be simulated for 10 times, and one time simulation lasts for 600 rounds, then the result is the average value of these 10 times simulation. In the energy model, Ene elec $=40 \mathrm{~nJ} / \mathrm{bit}, \mathrm{Ene}^{\text {amp }}=60 \mathrm{~nJ} / \mathrm{bit}$.

Our proposed RNSRA is then compared with WRP in [11] (weighted rendezvous planning) and proposed-II in [12]. The performance metrics used for the comparison are energy consumption, standard deviation of energy consumption and network lifetime.

In the simulation, we use standard deviation (SD) to measure the imbalance between the node's energy consumption, for example, a wider variation means that some parts of the network are more likely to exhaust their energy soon. The metric SD is calculated as follow:

$$
S D=\sqrt{\frac{\sum_{\operatorname{Num}(V)}^{i=1}\left(\text { ene }\left(v_{i}\right)-\mu\right)}{\operatorname{Num}(V)}}
$$

where ene $\left(v_{i}\right)$ is the energy consumption of node $v_{i}, V$ is the set of sensor nodes, $N u m(V)$ is the number of sensor nodes, and $\mu$ is the average energy consumption of sensor nodes.

\subsection{Evaluation of the experimental results}

The simulation results are shown in Figs. 4, 5 and 6. Fig. 4 presents the energy consumption ratio curve trend for these protocols. The RNSRA achieves $10 \%$ and more reduction in network energy consumption, as compared with proposed-II [11] and WRP [12], although their network energy consumption is rising as the sink speed is increased. The reason is that the RNSRA can select the RNs with better QoS parameters (e.g. more residual energy, better distance to other RNs and more neighbor nodes) to form the sink moving tour. Then it would construct a more reliable transmission environment to reduce the energy consumption of nodes. 


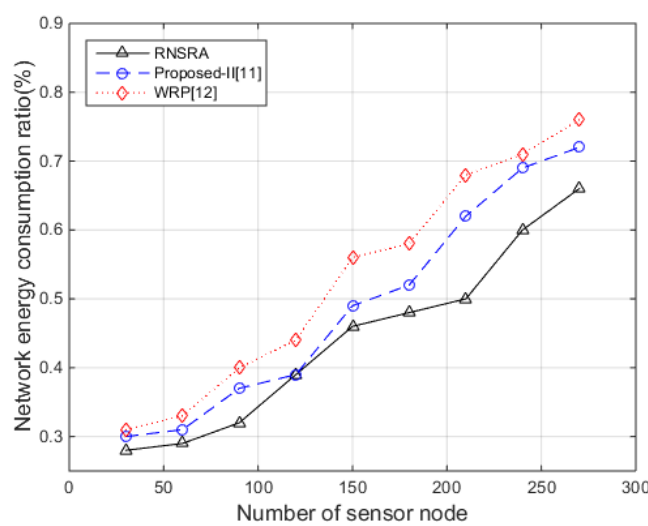

(a) Sink moving speed $2 \mathrm{~m} / \mathrm{s}$

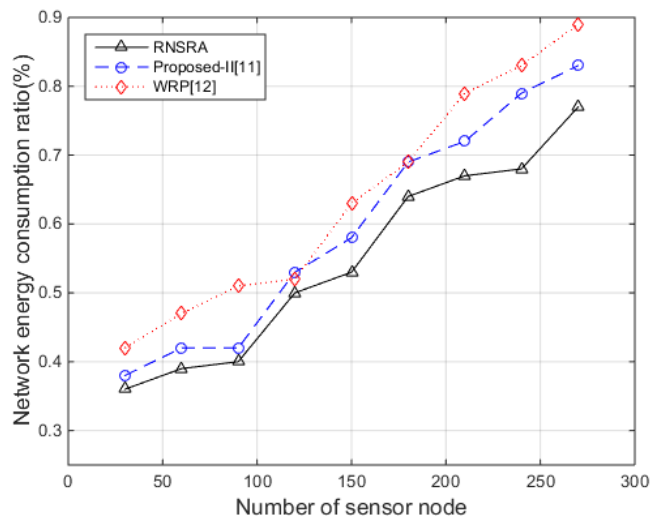

(b) Sink moving speed $4 \mathrm{~m} / \mathrm{s}$

Fig. 4. Network energy consumption for RNSRA, proposed-II [11] and WRP [12]

The ability of the three protocols uniformly distributing energy consumption is presented in Fig. 5, which indicates the standard deviation of energy consumption ratio to the sensor nodes number. We can observe that, our RNSRA distributes energy more uniformly than the other two protocols (5\% more than proposed-II, and $8 \%$ better than WRP). Comparing with proposed-II, RNSRA preferentially selects nodes with lower energy consumption rate and less hop count from the sink. After comparing with Figs 5 (a) and (b), we can observe that when the moving speed is increased from $2 \mathrm{~m} / \mathrm{s}$ to $4 \mathrm{~m} / \mathrm{s}$, the average SD value would increase by $10 \%$, that is because the increased speed of sink leads to the increase of network communication overhead, so that the energy consumption of some nodes becomes larger and the whole network energy consumption would be more unbalanced. However, the SD value of RNSRA is still lower than that of proposed-II and WRP, which reflects the advantage of network load balance performance of our proposed algorithm. 


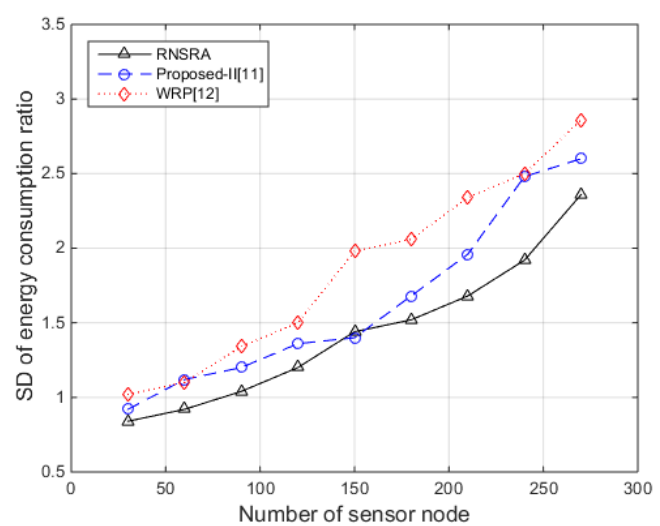

(a) Sink moving speed $2 \mathrm{~m} / \mathrm{s}$

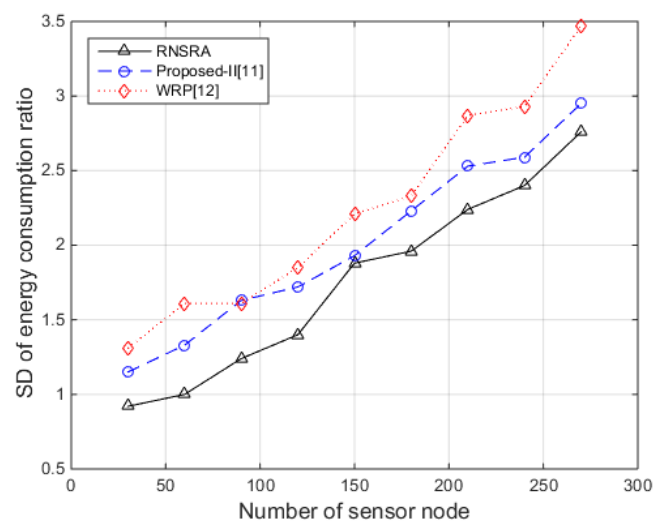

(b) Sink moving speed $4 \mathrm{~m} / \mathrm{s}$

Fig. 5. Standard deviation of energy consumption for RNSRA, proposed-II [11] and WRP [12]

We now evaluate the network lifetime performance of different protocols. As shown in Fig. 6, we observe that among the three protocols, RNSRA serves a reduction of $3 \%$ and $6 \%$ on network lifetime compared to proposed-II and WRP, that is because when the RN is exhausted, another optimal RN would be selected in our protocol, the network energy dispersion is balanced and it would take longer time for a node to exhaust energy. It can be seen from Figs 6 (a) and (b) that when the sink speed is doubled, the network lifetime of RNSRA is still better than that of proposed-II and WRP protocols. However, their survival time is reduced by $10 \%$ $\sim 20 \%$, it is because that the network energy consumption increases according to the higher sink moving speed, resulting in shorter node survival time. 


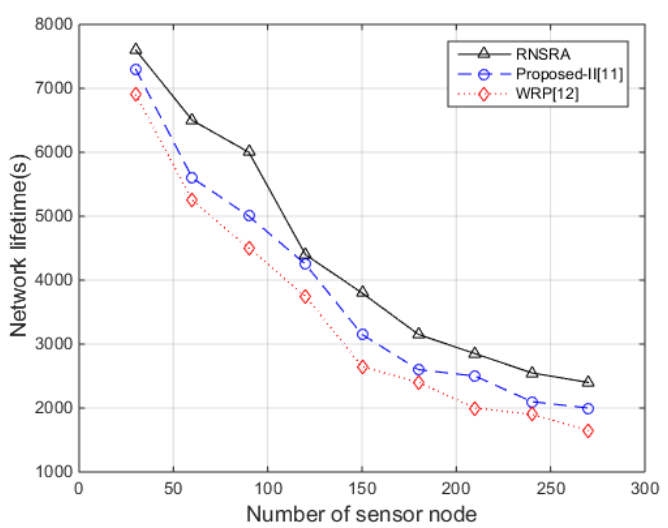

(a) Sink moving speed $2 \mathrm{~m} / \mathrm{s}$

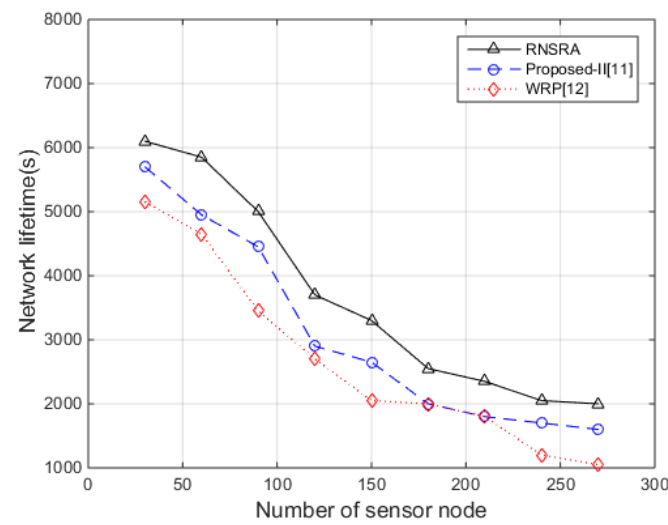

(b) Sink moving speed $4 \mathrm{~m} / \mathrm{s}$

Fig. 6. Network lifetime for RNSRA, proposed-II [11] and WRP [12]

\section{Conclusion}

This study presents a novel rendezvous node selection and routing algorithm for the mobile sink to visit the optimal RNs in the WSN, in order to plan an optimal moving tour for mobile sink. The proposed RNSRA would find suitable RNs with optimal factors for the mobile sink tour planning, and the other sensor nodes would use artificial bee colony optimization algorithm (ABC) to select optimal multi-hop path to forward data to the nearest RN. Such that the energy consumption of sensor nodes is minimized and uniform to avoid the problem of energy holes, while ensuring sensed data is gathered on time. More importantly, this paper demonstrated the applicability and the potential of RNSRA algorithm for solving RN selection optimization problems. The simulations show that the RNSRA could reduce energy consumption by $6 \%$ and increases network lifetime by $5 \%$, as compared with the other two protocols, they illustrate the novel capability of our method for efficiently balancing the network traffic load, and maintaining the network robustness against sink moving problem.

In the future, we will focus on the multi-mobile sink network and apply our method in heterogeneous WSN by taking advantage of the nodes with more computational power and storage capacity. We would also deploy the mobile sensor network with rendezvous node in the marine environment, in order to solve the marine monitoring problem [19]. 


\section{References}

[1] J. Aparicio, J. J. Echevarria, J. Legarda, “A software defined networking approach to improve the energy efficiency of mobile wireless sensor networks," KSII Transactions on Internet and Information Systems, vol. 11, no. 6, pp. 2848-2869, June, 2017. Article (CrossRef Link)

[2] K. Saleem, A. Derhab, J. Al-Muhtadi, M. A. Orgun, "Analyzing ant colony optimization based routing protocol against the hole problem for enhancing user's connectivity experience,” Computers in Human Behavior, vol. 51, pp. 1340-1350, 2015. Article (CrossRef Link)

[3] B. Zhang, Y. Wang, H. Wang, X. Guan, Z. Zhuang, "Tracking a duty-cycled autonomous underwater vehicle by underwater wireless sensor networks,” IEEE Access, vol. 5, pp.18016-18032, 2017. Article (CrossRef Link)

[4] Y. F. Hu, Y. S. Ding, K. R. Hao, H. Han, “An endocrine cooperative particle swarm optimization algorithm for routing recovery problem of wireless sensor networks with multiple mobile sinks, ” Information Sciences, vol. 300, pp. 100-113, 2015. Article (CrossRef Link)

[5] C. Tunca, S. Isik, M. Y. Donmez, and C. Ersoy, "Distributed mobile sink routing for wireless sensor networks: A survey,” IEEE Communications Surveys \& Tutorials, vol. 16, no. 2, pp. 877-897, 2014. Article (CrossRef Link)

[6] Y. S. Jiang, W. R. Shi, X. G. Wang and H. B. Li, “A distributed routing for wireless sensor networks with mobile sink based on the greedy embedding,” Ad Hoc Networks, vol. 20, pp. 150-162, 2014. Article (CrossRef Link)

[7] W. Cai, M. Zhang, "3D Dubins curves based path programming for mobile sink in underwater sensor networks,” Electronics Letters, 53(1) :48-50, 2016. Article (CrossRef Link)

[8] M. Abo-Zahhad, S. M. Ahmed, N. Sabor, and S. Sasaki, "Mobile sink-based adaptive immune energy efficient clustering protocol for improving the lifetime and stability period of wireless sensor networks,” IEEE Sensors Journal, vol. 15, no. 8, pp. 4576-4586, 2015. Article (CrossRef Link)

[9] J. Y. Chang, T. H. Shen, "An efficient tree-based power saving scheme for wireless sensor networks with mobile sink,” IEEE Sensors Journal, vol. 16, no. 20, pp. 7545-7557, 2016. Article (CrossRef Link)

[10] H. Salarian, C. Kwan-Wu; F. Naghdy, “An energy-efficient mobile-sink path selection strategy for wireless sensor networks,” IEEE Trans. Vehicular Technology, vol. 63, no. 5, pp. 2407-2419, 2014. Article (CrossRef Link)

[11] S. Mottaghi, M. R. Zahabi, "Optimizing LEACH clustering algorithm with mobile sink and rendezvous nodes,” AEU - International Journal of Electronics and Communications, vol. 69, no. 2, pp. 507-514, 2015. Article (CrossRef Link)

[12] B. Suh and S. Berber, "Rendezvous points and routing path-selection strategies for wireless sensor networks with mobile sink,” Electronics Letters, vol. 52, no. 2, pp. 167-169, 2016. Article (CrossRef Link)

[13] E. Lim, B. Kim and H. Min, “An energy efficient rendezvous node selection approach,” in Proc. of 2017 International Conference on Information Networking (ICOIN), pp. 423-425, 2017. Article (CrossRef Link)

[14] A. Sharifkhani, N. C. Beaulieu, "A mobile-sink-based packet transmission scheduling algorithm for dense wireless sensor networks," IEEE Trans. Vehicular Technology, vol. 58, no. 5, pp. 2509-2518, 2009. Article (CrossRef Link)

[15] R. Sugihara and R. Gupta, "Improving the data delivery latency in sensor networks with controlled mobility,” Distributed Computing in Sensor Systems, pp. 386-399, 2008. Article (CrossRef Link)

[16] R. S. Chang, S. H. Wang, S. L. Tsai, W. P. Yang, "Planning rendezvous using the Halin graph in wireless sensor networks,” Wireless Sensor Systems, IET, vol. 2, no. 3, pp. 222-229, 2012. Article (CrossRef Link)

[17] G. L. Xing, T. Wang, Z. H. Xie, and W. J. Jia, "Rendezvous planning in wireless sensor networks with mobile elements,” IEEE Trans. Mobile Computing, vol. 7, no. 12, pp. 1430-1443, Dec. 2008. Article (CrossRef Link) 
[18] Y. Z. Wu, T. Weise, R. Chiong, "Local search for the Traveling Salesman Problem: A comparative study," in Proc. of 2015 IEEE 14th International Conference on Cognitive Informatics \& Cognitive Computing (ICCI*CC2015), pp. 213 - 220, July, 2015. Article (CrossRef Link)

[19] M. Z. Abbas, K. A. Bakar, M. Ayaz, "Hop-by-hop dynamic addressing based routing protocol for monitoring of long range underwater pipeline," KSII Transactions on Internet and Information Systems, vol. 11, no. 2, pp. 731-763, Feb. 2017. Article (CrossRef Link)
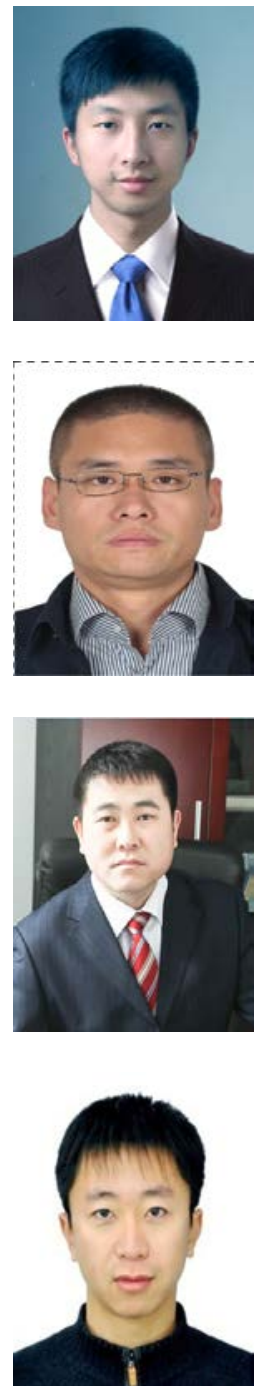

Yifan Hu received his $\mathrm{PhD}$, master and bachelor degrees at the Donghua University, Shanghai, China. He is currently an associate research fellow in Institute of Oceanographic Instrumentation, Qilu University of Technology(Shandong Academy of Sciences). His research interests include wireless sensor network for Internet of Things and intelligent computing.

Yi Zheng received his PhD degree at Beijing Institute of Technology, master degree at Shandong University of Science and Technology, and bachelor degree at Shandong University of Mining and Technology, Taian, China. He is currently an research fellow in Institute of Oceanographic Instrumentation, Qilu University of Technology(Shandong Academy of Sciences). His research interest is Machinery Manufacturing and Automation.

Xiaoming Wu received his $\mathrm{PhD}$ degree at Shandong University of Science and Technology, Qingdao, China; master and bachelor degree at Shandong University, Jinan, China. He is currently an associate research fellow in Shandong Computer Science Center (National Supercomputer Center in Jinan), Qilu University of Technology(Shandong Academy of Sciences). His research interest is mobile sensor network and information security.

Hailin Liu received his $\mathrm{PhD}$ and master degrees at the Ocean University of China, Qingdao, China, bachelor degree at the Qufu Normal University, Jining, China. He is currently an assistant research fellow in Institute of Oceanographic Instrumentation, Qilu University of Technology(Shandong Academy of Sciences). His research interests include Automatic control and UAV formation. 